\title{
An improved LC-MS/MS method for quantitative determination of ilaprazole and its metabolites in human plasma and its application to a pharmacokinetic study
}

\author{
Gan ZHOU ${ }^{1}$, Zhi-rong TAN ${ }^{1}$, Wei ZHANG ${ }^{1}$, Dong-sheng OU-YANG, Yao CHEN ${ }^{1}$, Dong GUO ${ }^{1}$, Ying-zi LIU ${ }^{1}$, Lan FAN ${ }^{1}$, Han-wu \\ $\mathrm{DENG}^{2, *}$ \\ ${ }^{1}$ Pharmacogenetics Research Institute, Institute of Clinical Pharmacology, Central South University, Changsha 410078, China; ${ }^{2}$ Depart- \\ ment of Pharmacology, School of Pharmaceutical Sciences, Central South University, Changsha 410078, China
}

\begin{abstract}
Aim: To improve and validate an analytical method based on liquid chromatography and electrospray ionization tandem mass spectrometry (LC-ESI-MS/MS) for the quantitative measurement of ilaprazole and its two metablites in human plasma.

Methods: Separation of analytes and the internal standard (IS), omeprazole, was performed on a Thermo HyPURITY C18 column $(150 \times 2.1 \mathrm{~mm}, 5 \mu \mathrm{m})$ with a mobile phase consisting of $10 \mathrm{mmol} / \mathrm{L}$ ammonium formate water-acetonitrile solution (50:50, $v / v)$ at a flow rate of $0.25 \mathrm{~mL} / \mathrm{min}$. The API4000 triple quadruple mass spectrometer was operated in multiple reactions monitoring mode via positive electrospray ionization interface using the transition $\mathrm{m} / \mathrm{z} 367.2 \rightarrow \mathrm{m} / \mathrm{z} 184.0$ for ilaprazole, $\mathrm{m} / \mathrm{z} 383.3 \rightarrow \mathrm{m} / \mathrm{z} 184.1$ for ilaprazole sulfone, $\mathrm{m} / \mathrm{z} 351.2 \rightarrow \mathrm{m} / \mathrm{z} 168.1$ for ilaprazole thiol ether and $\mathrm{m} / \mathrm{z} 346.2 \rightarrow \mathrm{m} / \mathrm{z} 198.0$ for omeprazole.

Results: The method was linear over the concentration range of $0.23-2400.00 \mathrm{ng} / \mathrm{mL}$ for ilaprazole, $0.05-105.00 \mathrm{ng} / \mathrm{mL}$ for ilaprazole thiol ether and $0.06-45.00 \mathrm{ng} / \mathrm{mL}$ for ilaprazole sulfone. The intra- and inter-day precisions were all less than $15 \%$ in terms of relative standard deviation (RSD), and the accuracy was within 15\% in terms of relative error (RE) for ilaprazole, ilaprazole sulfone and ilaprazole thiol ether. The lower limit of quantification (LLOQ) was identifiable and reproducible at $0.23,0.05 \mathrm{and} 0.06 \mathrm{ng} / \mathrm{mL}$ with acceptable precision and accuracy for ilaprazole, ilaprazole sulfone and ilaprazole thiol ether, respectively.

Conclusion: The validated method offered sensitivity and a wide linear concentration range. This method was successfully applied for the evaluation of the pharmacokinetics of ilaprazole and its two metabolites after single oral doses of 5 mg ilaprazole to 12 healthy Chinese volunteers.
\end{abstract}

Keywords: ilaprazole; ilaprazole sulfone; ilaprazole thiol ether; metabolite; LC-MS/MS; electrospray ionization; pharmacokinetics

Acta Pharmacologica Sinica (2009) 30: 1330-1336; doi: 10.1038/aps.2009.120; published online 24 August 2009

\section{Introduction}

Ilaprazole[-[(4-methoxy-3-methyl-pyridin-2-yl)methylsulfinyl]-6-pyrrol-1-yl-1H-benzoimidazole, Figure 1], a substituted benzimidazole, is a new candidate drug that is an $\mathrm{H}^{+} / \mathrm{K}^{+}$-ATPase inhibitor designed for the treatment of gastric ulcers $^{[1,2]}$. Ilaprazole was under development by IIYang Pharmacy Co (Seoul, Korea) and has been proven by a series of animal studies to be a potent and safety antiulcer agent. SeungWoon Myung et al found two metabolites of ilaprazole using LC-MS/MS in rat plasma, the major one being ilaprazole sulfone (Figure 1$)^{[3]}$. Recently, a new metabolite of ilaprazole,

\footnotetext{
* To whom correspondence should be addressed.

E-mail denghanwu@163.com

Received 2009-04-07 Accepted 2009-07-03
}

ilaprazole thiol ether, was identified (Figure 1).

Quantification of drugs in biological matrices by liquid chromatography/tandem mass spectrometry (LC/MS/MS) is becoming an increasingly common technology today due to the improved sensitivity and selectivity of these methods. Li et al reported that ilaprazole was metabolized by CYP3A5 and CYP2C9 in Chinese healthy subjects using a LC-MS/MS method to determine ilaprazole and ilaprazole sulfone concentrations ${ }^{[4]}$. We now report an improved LC/MS/MS method and have validated the procedure for the simultaneous detection and quantification of ilaprazole, ilaprazole sulfone and ilaprazole thiol ether in human plasma. Furthermore, this method is capable of quantifying ilaprazole, ilaprazole sulfone and ilaprazole thiol ether at concentrations of $0.23 \mathrm{ng} / \mathrm{mL}, 0.05$ and $0.06 \mathrm{ng} / \mathrm{mL}$, respectively. At the same time, it is expected 


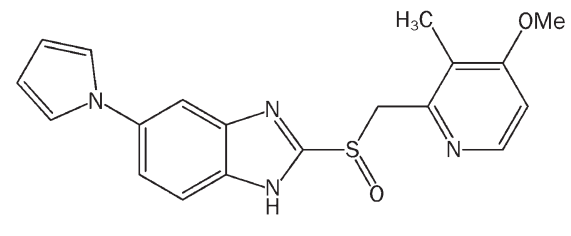

Ilaprazole

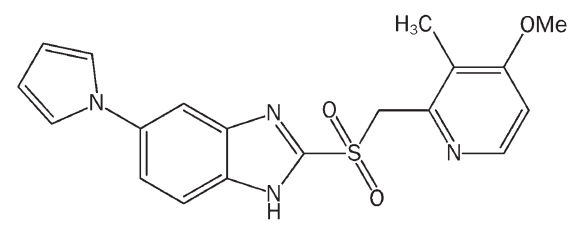

Ilaprazole sulfone

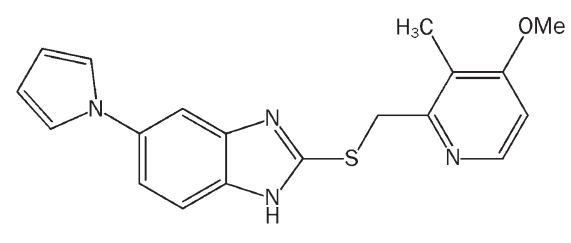

Ilaprazole thiol ether

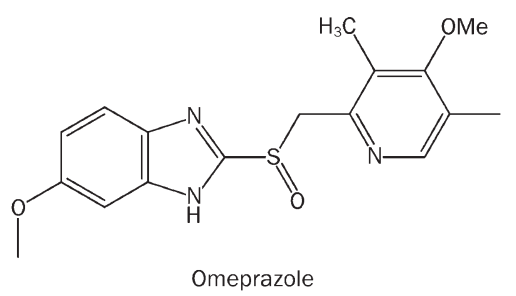

Figure 1. Chemical structure of ilaprazole, ilaprazole sulfone, ilaprazole thiol ether and omeprazole.

that this method would be efficient in analyzing a large number of plasma samples obtained for pharmacokinetic, bioavailability or bioequivalence studies after administration of therapeutic doses of ilaprazole.

\section{Materials and methods Materials and reagents}

Ilaprazole, ilaprazole sulfone and ilaprazole thiol ether standards were obtained from the Livzon Pharmaceutical Group Inc (purity: 99.1\% Zhuhai, China); omeprazole standards were obtained from Sigma Company (purity: 99.5\%, USA). Ilaprazole tablets were kindly supplied from Livzon Pharmaceutical Group Inc (Zhuhai, China). HPLC-grade acetonitrile; methanol and methyl tert-butyl ether (MTBE) were purchased from Dikma Comp (Guangzhou, China). HPLC-grade water was from a Milli-Q system (Millipore, USA). All other reagents were of analytical grade.

\section{Instruments}

The instrumentation system used in this study consisted of an Agilent 1200 series RPLC (vacuum degasser, binary pump, and thermo stated column compartment) from Agilent Tech- nologies (Palo Alto, CA, USA), and an API4000 triple quadruple mass spectrometer by Applied Biosystems (Foster City, CA, USA) with an electrospray (ESI) Turbo VTM Ion Source. A HyPURITY C18 $(150 \times 2.1 \mathrm{~mm}, 5 \mu \mathrm{m})$ column by ThermoHypersil Keystone (Bellefonte, PA) was used. The data was collected and processed using Analyte 1.4.2 software from Applied Biosystems (Foster City, CA, USA).

\section{Chromatographic conditions}

The separation was performed on a Thermo HyPURITY C18 column $(150 \mathrm{~mm} \times 2.1 \mathrm{~mm}, 5 \mu \mathrm{m})$. The mobile phase, consisting of a solution of $10 \mathrm{mmol} / \mathrm{L}$ ammonium formate water solution-acetonitrile $(50: 50, v / v)$, was pumped at a flow rate of $0.25 \mathrm{~mL} / \mathrm{min}$. Prior to the analysis, the column was equilibrated with the mobile phase at a flow rate of $0.25 \mathrm{~mL} / \mathrm{min}$ for about $15 \mathrm{~min}$. During the analysis, $10 \mu \mathrm{L}$ of sample was injected by the auto sampler and carried on the analyst column by the mobile phase.

\section{Mass spectrometer conditions}

The API4000 triple quadruple mass spectrometer was operated under the positive electrospray ionization mode $\left(\mathrm{ESI}^{+}\right)$. The mass spectrometer was tuned by infusion of ilaprazole and its two metabolites $(1.0 \mu \mathrm{g} / \mathrm{mL})$ and omeprazole $(1.0 \mu \mathrm{g} / \mathrm{mL})$ in the mobile phase at a flow rate of $10 \mu \mathrm{L} / \mathrm{min}$ with a syringe pump (Harvard Apparatus, South Natick, MA, USA). The tandem mass spectrometer was tuned to monitor the $m / z 367.2$ $\rightarrow m / z 184.0$ for ilaprazole, $m / z 383.3 \rightarrow m / z 184$.1 for ilaprazole sulfone, $m / z 351.2 \rightarrow m / z 168.1$ for ilaprazole thiol ether and $m / z$ $346.2 \rightarrow m / z 198.0$ for omeprazole, respectively, using positive electrospray ionization ${ }^{[5]}$. The spectra and the proposed patterns of ilaprazole, ilaprazole sulfone, ilaprazole thiol ether and omeprazole fragmentation, as well as a summary of the adjusted MS conditions and the compound-specific MSparameters, are presented in Figure 2 and Table 1.

Preparation of stock solutions, calibration standard and quality control samples (QC)

Stock solutions of ilaprazole, ilaprazole sulfone and ilaprazole thiol ether at the concentrations of $2.40 \mathrm{mg} / \mathrm{mL}, 4.50 \mathrm{mg} / \mathrm{mL}$, and $1.05 \mathrm{mg} / \mathrm{mL}$, respectively, were prepared in methanol. The internal standard (omeprazole) was also prepared as a stock solution $(2.80 \mathrm{mg} / \mathrm{mL})$ in methanol and was further diluted with $10 \mathrm{mmol} / \mathrm{L}$ ammonium formate water solution to give a concentration of $280 \mathrm{ng} / \mathrm{mL}$ and used for all analyses. Serial calibration curve samples at concentrations of 0.23 , $0.47,3.75,15.00,60.00,300.00,600.00,1200.00$, and 2400.00 $\mathrm{ng} / \mathrm{mL}$ of ilaprazole, $0.06,0.18,0.70,2.81,5.63,11.30,45.00$ $\mathrm{ng} / \mathrm{mL}$ of ilaprazole sulfone, and $0.05,0.10,0.41,1.64,6.56$, 26.30 and $105.00 \mathrm{ng} / \mathrm{mL}$ of ilaprazole thiol ether were freshly prepared by serially diluting a stock solution with drug-free plasma. The quality control (QC) samples were prepared in the same way at concentrations of 0.23 (LLOQ), 0.47 (low), 15.00 (medium) and $1200.00 \mathrm{ng} / \mathrm{mL}$ (high) of ilaprazole, 0.06 (LLOQ), 0.18 (low), 2.81 (medium) and $45.00 \mathrm{ng} / \mathrm{mL}$ (high) of ilaprazole sulfone, 0.05 (LLOQ), 0.10 (low), 1.64 (medium) and 

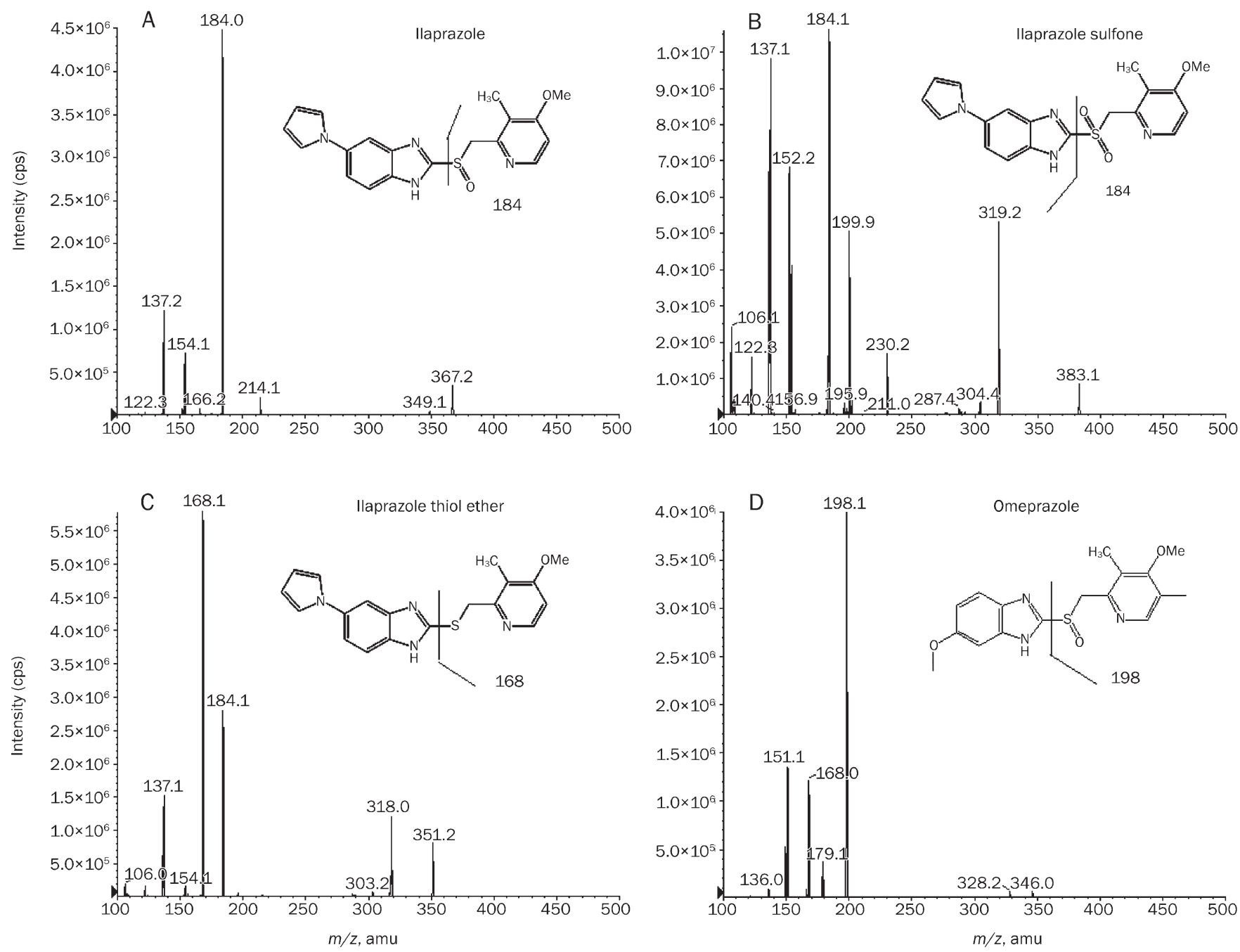

Figure 2. Production mass spectrum of ila prazole, ilaprazole sulfone, ilaprazole thiol ether, and omeprazole.

$105.00 \mathrm{ng} / \mathrm{mL}$ (high) of ilaprazole thiol ether. All plasma samples were stored at $-40{ }^{\circ} \mathrm{C}$. Fifty microliters of IS $(280 \mathrm{ng} / \mathrm{mL})$ was added to $0.2 \mathrm{~mL}$ of calibration curve samples and quality control samples. The further processing of both the calibration curve samples and the quality control samples were the same as described in the following section (section 2.6) for collection and preparation of the samples. All standard stock solutions were prepared once a month and stored at $-40{ }^{\circ} \mathrm{C}^{[6]}$.

\section{Sample preparation}

All frozen human plasma samples were thawed at room temperature. In order to perform sample extraction, $0.2 \mathrm{~mL}$ of the sample (human plasma) was added in a $1.5 \mathrm{~mL}$ eppendorf tube (EP), and $100 \mu \mathrm{L}$ of $50 \mathrm{mmol} / \mathrm{L}$ ammonium formate water solution plus $50 \mu \mathrm{L}$ of $280 \mathrm{ng} / \mathrm{mL}$ omeprazole standard solution was added; $0.6 \mathrm{~mL}$ of MTBE was subsequently added. The extraction was then performed by vortex-mixing for 2 min, followed by centrifugation of the mixture at11 $500 \times \mathrm{g}$ for $10 \mathrm{~min}$. The organic phase was placed into another EP tube, and evaporated to dryness under a stream of $\mathrm{N}_{2}$. The residue was reconstituted in $200 \mu \mathrm{L}$ of mobile phase solution and10 $\mu \mathrm{L}$ of the mixture was injected into the LC-MS/MS system.

\section{Clinical trial}

The developed LC-MS-MS method was applied to determine the plasma concentrations of ilaprazole from a clinical trial study in which 12 healthy male Chinese subjects were enrolled. The subjects ranged in age from 19 to 27 years

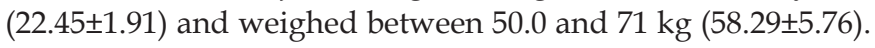
The pharmacokinetic study was approved by the medical Ethics Committee of Hunan Xiangya Hospital. Informed consent was obtained from all subjects after explaining the aims and risks of the study. The doses of ilaprazole chosen for this study were based on findings from preclinical and early clinical studies. Twelve volunteers continuously received 10 mg ilaprzole enteric-coated tablet for 5 days. Blood samples were collected on the fifth day. An indwelling cannula was placed in the other arm for blood sampling. Before sampling, 
Table 1. MS conditions for ilaprazole, ilaprazole sulfone, ilaprazole thiol ether.

\begin{tabular}{|c|c|c|c|c|c|}
\hline \multicolumn{3}{|c|}{ Mass spectrometer } & \multicolumn{3}{|c|}{ API4000 } \\
\hline \multicolumn{3}{|l|}{ Interface } & \multicolumn{3}{|c|}{ Electrospray } \\
\hline \multicolumn{3}{|l|}{ Polarity } & \multicolumn{3}{|c|}{ Polaritive } \\
\hline \multicolumn{3}{|l|}{ Scan type } & \multicolumn{3}{|l|}{ MRM } \\
\hline \multicolumn{3}{|l|}{ Resolution } & \multicolumn{3}{|c|}{ Q1-unit resolution } \\
\hline \multicolumn{3}{|c|}{ Curtain gas (CUR) } & \multicolumn{3}{|c|}{ Q3-unit resolution } \\
\hline \multicolumn{3}{|c|}{ Collision gas (CAD) } & \multicolumn{3}{|c|}{6} \\
\hline \multicolumn{3}{|c|}{ IonSpray voltage (IS) } & \multicolumn{3}{|l|}{4500} \\
\hline \multicolumn{3}{|c|}{ Temperature (TEM) } & \multicolumn{3}{|c|}{$500^{\circ} \mathrm{C}$} \\
\hline \multicolumn{3}{|c|}{ Ion source gas 1 (GS 1) } & \multicolumn{3}{|l|}{45} \\
\hline \multicolumn{3}{|c|}{ Ion source gas 2 (GS 2) } & \multicolumn{3}{|l|}{65} \\
\hline \multicolumn{3}{|c|}{ Solvent split ratio } & \multicolumn{3}{|l|}{ None } \\
\hline \multirow[t]{2}{*}{ Compound } & \multicolumn{2}{|c|}{$\begin{array}{c}\text { Mass to charge } \\
\text { ratio }(\mathrm{m} / \mathrm{z})\end{array}$} & \multirow[t]{2}{*}{ DP } & \multirow[t]{2}{*}{$\mathrm{CE}$} & \multirow{2}{*}{$\begin{array}{r}\text { Dwell } \\
\text { time } \\
\text { (ms) }\end{array}$} \\
\hline & Q1 (amu) & Q3 (amu) & & & \\
\hline Ilaprazole & 367.2 & 184.0 & 58 & 20 & 200 \\
\hline Ilaprazole sulfone & 383.3 & 184.1 & 80 & 39 & 200 \\
\hline Ilaprazole thiol ether & 351.2 & 168.1 & 90 & 35 & 200 \\
\hline Omeprazole (ISTD) & 346.2 & 198.0 & 98 & 22 & 200 \\
\hline
\end{tabular}

about $0.5 \mathrm{~mL}$ of blood was discarded followed by collection of the subsequent $5 \mathrm{~mL}$ blood samples in heparinized tubes at the following times on the days of the pharmacokinetic measurements: immediately prior to drug administration $(0 \mathrm{~h})$, and at $0.5,1,1.5,2,3,4,6,8,12,24,36,48,60$, and $72 \mathrm{~h}$ after drug administration. The blood samples were centrifuged at $2000 \times g\left(4{ }^{\circ} \mathrm{C}\right)$ for $10 \mathrm{~min}$ to separate the plasma fractions and the plasma samples were stored at $-40^{\circ} \mathrm{C}$ until analysis.

Calculation of the pharmacokinetic parameters was performed by non-compartmental assessment of data using the computer program WinNonlin (v 5.0.1, Pharsight, Mountain View, CA, USA). The maximum plasma concentrations $\left(C_{\max }\right)$ and their time of occurrence $\left(T_{\max }\right)$ were both obtained directly from the measured data. The area under the plasma concentration-time curve from time zero to the time of the last measurable concentration $\left(\mathrm{AUC}_{0-\mathrm{t}}\right)$ was calculated by the linear trapezoidal method. The terminal elimination rate constant $\left(k_{e}\right)$ was estimated by log-linear regression of concentrations observed during the terminal phase of elimination, and the corresponding elimination half-life $\left(t_{1 / 2}\right)$ was then calculated as $0.693 / k_{e}$.

\section{Results}

\section{Extraction method}

The purpose of this study was to develop a simple and inexpensive extraction method that could be readily implemented in monitoring labs. Therefore, our work focused on the use of liquid-liquid extraction. Hexane: ethylene chloride, MTBE, chloroform ${ }^{[4]}$, dichloromethane and ethyl acetate were evaluated as extraction solvents under neutral conditions with 100 $\mathrm{mmol} / \mathrm{L}$ ammonium formate. The best recovery was observed
Table 2. Representative intraday precision and accuracy data for the determination of ilaprazole, ilaprazole sulfone and ilaprazole thiol ether in five different lots of plasma as assessed by the replicate $(n=5)$.

\begin{tabular}{|c|c|c|c|c|}
\hline Drug & $\begin{array}{c}\text { Nominal } \\
\text { concentration } \\
(\mathrm{ng} / \mathrm{mL})\end{array}$ & $\begin{array}{c}\text { Mean assayed } \\
\text { concentration } \\
(\mathrm{ng} / \mathrm{mL}, n=5)\end{array}$ & $\begin{array}{c}\text { Accuracy } \\
(\%)^{\mathrm{a}}\end{array}$ & $\begin{array}{c}\text { Precision } \\
(\%)^{\mathrm{b}}\end{array}$ \\
\hline \multirow[t]{9}{*}{ Ilaprazole } & 0.23 & 0.24 & 103.4 & 5.6 \\
\hline & 0.47 & 0.48 & 102.1 & 4.2 \\
\hline & 3.75 & 3.84 & 102.4 & 4.1 \\
\hline & 15.00 & 16.25 & 108.3 & 6.3 \\
\hline & 60.00 & 59.62 & 99.4 & 4.5 \\
\hline & 300.00 & 325.64 & 108.5 & 5.9 \\
\hline & 600.00 & 619.52 & 103.3 & 3.2 \\
\hline & 1200.00 & 1125.68 & 93.8 & 6.1 \\
\hline & 2400.00 & 2389.65 & 99.6 & 3.1 \\
\hline \multirow[t]{7}{*}{ Ilaprazole sulfone } & 0.06 & 0.06 & 104.5 & 6.2 \\
\hline & 0.18 & 0.18 & 102.3 & 4.1 \\
\hline & 0.70 & 0.76 & 107.5 & 5.1 \\
\hline & 2.81 & 2.95 & 105.0 & 3.9 \\
\hline & 5.63 & 5.81 & 103.2 & 4.7 \\
\hline & 11.30 & 11.12 & 98.4 & 6.5 \\
\hline & 45.00 & 44.95 & 99.9 & 1.2 \\
\hline \multirow[t]{7}{*}{ Ilaprazole thiol ether } & 0.05 & 0.06 & 117.2 & 9.5 \\
\hline & 0.10 & 0.11 & 107.8 & 7.4 \\
\hline & 0.41 & 0.45 & 109.8 & 3.8 \\
\hline & 1.64 & 1.74 & 106.1 & 4.2 \\
\hline & 6.56 & 6.32 & 96.3 & 6.9 \\
\hline & 26.30 & 25.98 & 98.8 & 3.5 \\
\hline & 105.00 & 107.89 & 102.8 & 2.4 \\
\hline
\end{tabular}

${ }^{a}$ Expressed as [(mean observed concentration)/(nominal concentration) $] \times 100$.

${ }^{\mathrm{b}}$ Coefficient of variation.

with chloroform and MTBE as the extraction solvent. Since it can be easily evaporated, MTBE was selected as the extraction solvent.

\section{Assay validation}

Assay validation was performed in accordance with current guidelines $^{[7,8]}$

\section{Representative chromatogram}

Representative chromatograms of a control double blank sample, a control single blank sample containing $280 \mathrm{ng} / \mathrm{mL}$ ISTD, and a $0.23 \mathrm{ng} / \mathrm{mL}, 0.06$ and $0.05 \mathrm{ng} / \mathrm{mL}$ ilaprazole, ilaprazole sulfone and ilaprazole thiol ether, respectively, plasma standard (LLOQ) are shown in Figure 3A-3C .

\section{Intraday variability}

An assessment of intraday variability was conducted by analyzing standard curve samples in five different lots of human control plasma over the calibration range of $0.23-2400.00 \mathrm{ng} /$ $\mathrm{mL}, 0.06-45.00 \mathrm{ng} / \mathrm{mL}, 0.05-105.00 \mathrm{ng} / \mathrm{mL}$ for ilaprazole, 

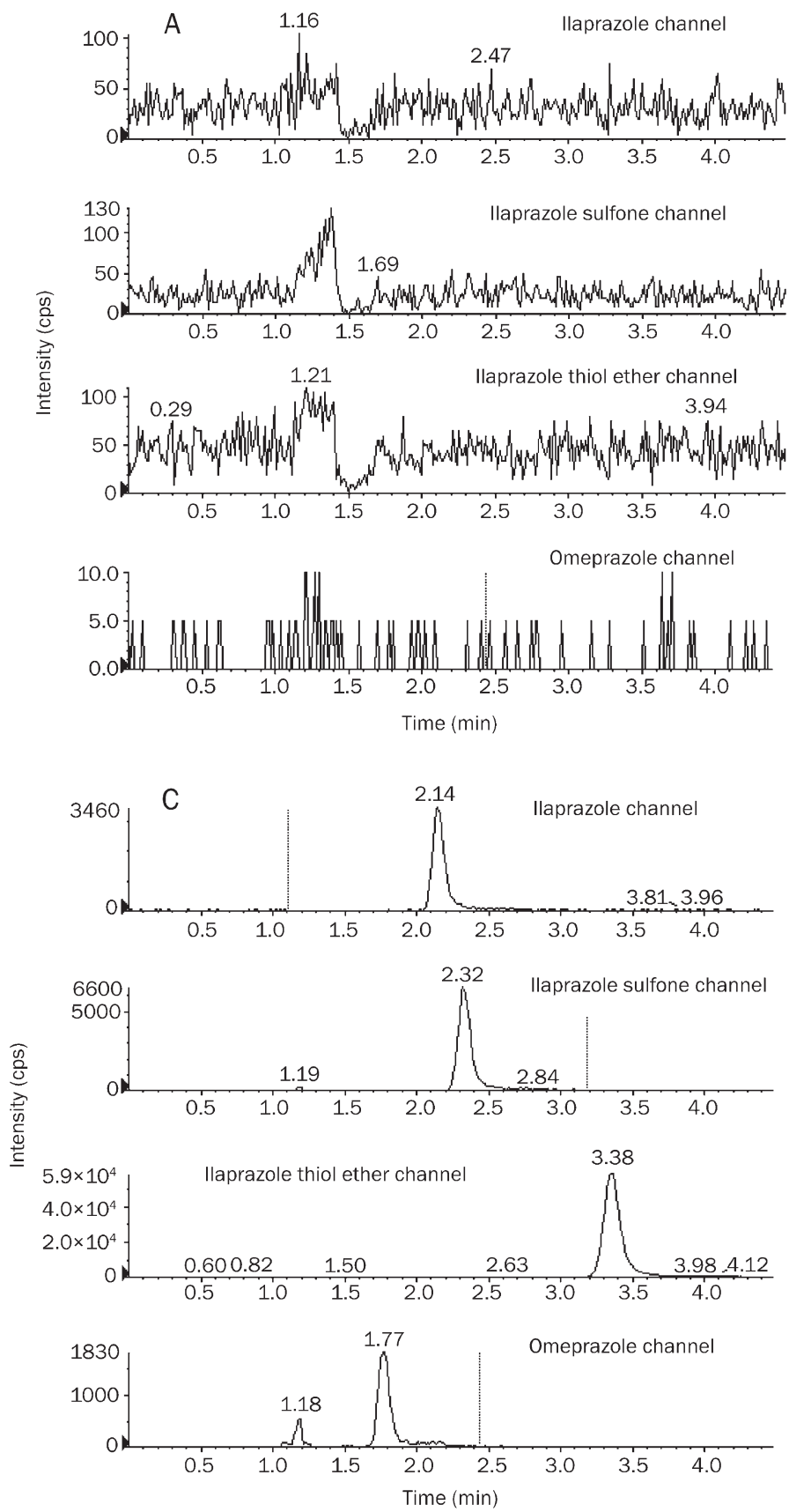

ilaprazole sulfone and ilaprazole thiol ether, respectively. The resulting precision and accuracy data are presented in Table 2. The coefficient of variation of the assay was $\leq 9.5$ for all concentrations within the standard curve range, while the assay accuracy was $93.8 \%-117.2 \%$ of nominal.

\section{Quality control samples}

Quality control (QC) samples containing concentrations of 0.23 $\mathrm{ng} / \mathrm{mL}$ (LLOQ), $0.47 \mathrm{ng} / \mathrm{mL}$ (low), $15.00 \mathrm{ng} / \mathrm{mL}$ (medium) and $1200.00 \mathrm{ng} / \mathrm{mL}$ (high) of ilaprazole, $0.06 \mathrm{ng} / \mathrm{mL}$ (LLOQ), $0.18 \mathrm{ng} / \mathrm{mL}$ (low), $2.81 \mathrm{ng} / \mathrm{mL}$ (medium) and $45.00 \mathrm{ng} / \mathrm{mL}$ (high) of ilaprazole sulfone, $0.05 \mathrm{ng} / \mathrm{mL}$ (LLOQ), $0.10 \mathrm{ng} /$ $\mathrm{mL}$ (low), $1.64 \mathrm{ng} / \mathrm{mL}$ (medium) and $105.00 \mathrm{ng} / \mathrm{mL}$ (high) of
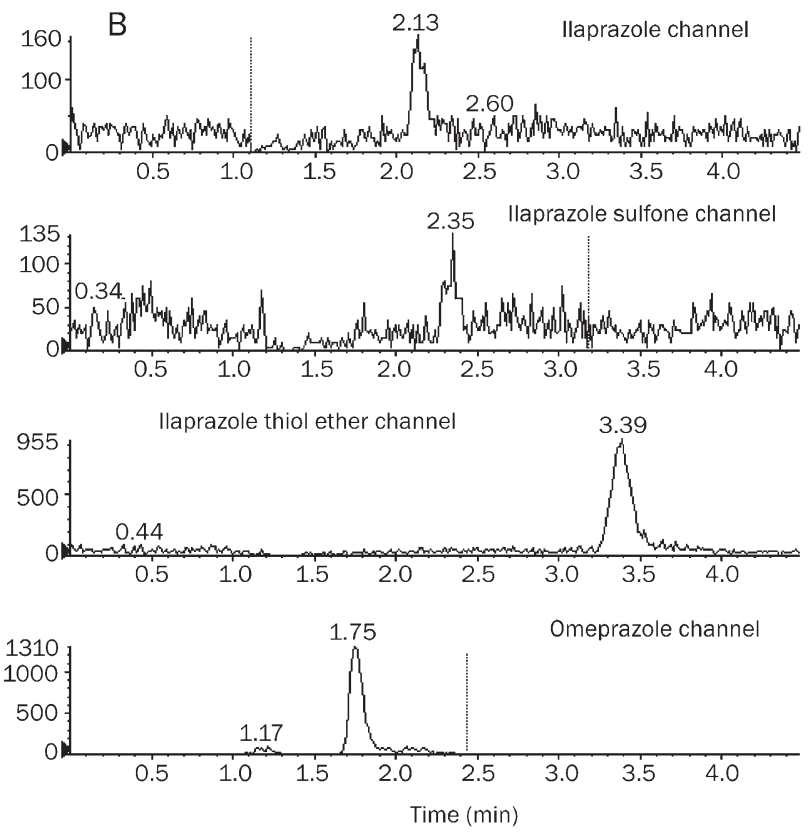

Figure 3. (A) Representative chromatogram of a control plasma double blank sample. (B) Representative chromatogram of an extracted $0.23 \mathrm{ng} / \mathrm{mL}$ ilaprazole, $0.05 \mathrm{ng} / \mathrm{mL}$ ilaprazole sulfone and $0.06 \mathrm{ng} / \mathrm{mL}$ ilaprazole thiol ether standard in control plasma. (C) Representative chromatogram from a clinical subject collected $3 \mathrm{~h}$ after dosing with $10 \mathrm{mg}$ ilaprazole. ilaprazole thiol ether were used. Initial within-day analysis of the QC samples was within 8.3 of nominal with a CV of 9.5 or below (Table 3).

\section{Inter-day variability of quality control samples}

Robustness of the described method was demonstrated through inter-day $(n=15)$ analysis of QC samples over a 2-month study span. The average QC accuracy was within $6.52 \%$ of nominal and the precision was $4.25 \%$ or below.

\section{Extraction recovery and assessment of the Matrix effect on} ionization

Extraction recovery and the effect of the sample matrix on 
Table 3. Initial within-day analysis of ilaprzole, ilaprazole sulfone, ilaprazole thiol ether quality control samples.

\begin{tabular}{llrrrr}
\hline Drug & $\begin{array}{c}\text { Concentration } \\
(\mathrm{ng} / \mathrm{mL})\end{array}$ & $\begin{array}{c}\text { Initial } \\
\text { mean } \\
(n=5)\end{array}$ & $\begin{array}{r}\text { Standard } \\
\text { deviation }\end{array}$ & $\begin{array}{c}\text { Preci- } \\
\text { sion } \\
(\%)^{\mathrm{a}}\end{array}$ & $\begin{array}{c}\text { Accuracy } \\
(\%)^{\mathrm{b}}\end{array}$ \\
\hline Ilaprazole & Low QC (0.468) & 0.51 & 0.23 & 8.91 & 108.97 \\
& Middle QC (15) & 14.54 & 1.25 & 7.52 & 96.93 \\
& High QC (1200) & 1128.25 & 3.61 & 1.25 & 94.02 \\
Ilaprazole & Low QC (0.176) & 0.19 & 0.12 & 10.23 & 107.95 \\
sulfone & Middle QC (2.81) & 2.65 & 0.68 & 6.23 & 94.31 \\
& High QC (45.0) & 43.68 & 2.36 & 3.26 & 97.07 \\
Ilaprazole & Low QC (0.10) & 0.11 & 0.11 & 12.36 & 107.84 \\
thiol ether & Middle QC (1.64) & 1.59 & 0.75 & 6.89 & 96.95 \\
& High QC (105) & 102.35 & 4.52 & 3.74 & 97.48 \\
\hline
\end{tabular}

${ }^{\mathrm{a} C o e f f i c i e n t}$ of variation.

${ }^{b}$ Expressed as [(mean observed concentration)/(nominal concentration) $] \times 100$.

ionization were evaluated for ilaprazole, ilaprazole sulfone, ilaprazole thiol ether using standard samples prepared at three QC concentrations in five different individual human plasma samples. Recovery of the extraction was determined by comparing the absolute peak areas of the standards in human plasma extracted as described to control plasma extracted in the same manner and then spiked post extraction with the same concentration of the drug and internal standard. Matrix enhancement/suppression of ionization was evaluated by comparing the absolute peak areas of control plasma extracted and then spiked with a known amount of drug, to approximate standards injected directly in the same reconstitution solvent. Results are shown in Table 4. Based on the intra-day precision results that were obtained using six different lots of control plasma, a relative matrix effect was not expected to affect the assay (Table 4). The lack of a relative matrix effect is further illustrated in Table 5, where an examination of the slopes of the standard curves prepared from five different lots of control plasma shows a precision of 5.086, 11.731, and 5.205 for ilaprzole, ilaprzole sulfone and ilaprazole thiol ether, respectively.

\section{Stability}

This method assessed the freeze-thaw, room temperature, processed sample stability, short-term and long-term stability of ilaprazole and its metabolites. The results showed that the ilaprazole and its two metabolites were stable in all of the above experimental conditions.

\section{Clinical sample analysis}

Human plasma samples were analyzed following administration of oral $10 \mathrm{mg}$ doses of ilaprazole. A representative postdose chromatogram from $3 \mathrm{~h}$ after administration of a $10 \mathrm{mg}$ dose is shown in Figure 3C. Mean ( $n=12$ subjects) plasma
Table 4. Extraction recovery and assessment of absolute matrix effects on ionization during the determination of ilaprazole, ilaprazole sulfone, ilaprazole thiol ether in five different lots of human plasma.

\begin{tabular}{lccc}
\hline Drug & $\begin{array}{c}\text { Standard } \\
\text { concentration in } \\
\text { plasma }(\mathrm{ng} / \mathrm{mL})\end{array}$ & $\begin{array}{c}\text { Mean } \\
\text { extraction } \\
\text { recovery } \\
(\%)(n=5)^{\mathrm{a}}\end{array}$ & $\begin{array}{c}\text { Mean } \\
\text { absolute } \\
\text { matrix effect } \\
(\%)(n=5)^{\mathrm{b}}\end{array}$ \\
\hline Ilaprazole & 0.468 & 85.6 & 91.2 \\
Ilaprazole sulfone & 15 & 82.8 & 90.6 \\
& 1200 & 83.4 & 88.9 \\
Ilaprazole thiol ether & 0.176 & 81.0 & 92.3 \\
& 2.81 & 82.3 & 96.5 \\
& 45 & 78.9 & 91.2 \\
Omeprazole (ISTD) & & & \\
& 1.64 & 75.6 & 96.2 \\
& 105 & 79.5 & 90.5 \\
& 280 & 85.6 & 92.5 \\
\hline
\end{tabular}

${ }^{a}$ Extraction recovery was calculated by dividing the mean peak area of analyte spiked before extraction by the respective mean peak area of analyte spiked into extracts of control plasma and multiplying by 100 .

${ }^{\mathrm{b}}$ Matrix effect was calculated by dividing the mean peak area of analyte spiked into control plasma extracts by the mean peak area of the neat analyte standard and multiplying by 100 .

${ }^{c}(n=15)$.

Table 5. Slopes of standard curves in five different lots of control plasma (relative matrix effect).

\begin{tabular}{lccc}
\hline Control plasma lot & Ilaprazole & $\begin{array}{c}\text { Slopes } \\
\text { Ilaprazole } \\
\text { sulfone }\end{array}$ & $\begin{array}{r}\text { Ilaprazole } \\
\text { thiol ether }\end{array}$ \\
\hline A & 0.693 & 0.296 & 14.200 \\
B & 0.758 & 0.258 & 13.256 \\
C & 0.685 & 0.358 & 15.289 \\
D & 0.664 & 0.315 & 14.125 \\
E & 0.715 & 0.314 & 14.628 \\
Mean & 0.703 & 0.308 & 14.300 \\
Standard deviation & 0.036 & 0.036 & 0.744 \\
Precision (\%) & 5.086 & 11.731 & 5.205 \\
\hline
\end{tabular}

concentration $v$ s time profiles of ilaprazole, ilaprazole sulfone, and ilaprazole thiol ether after continuous oral administration of $10 \mathrm{mg}$ dose are shown in Figure 4.

\section{Discussion and conclusion}

The LC-MS-MS method had been reported in the literature ${ }^{[3,4]}$; however, this is the first time that the simultaneous detection of ilaprazole, ilaprazole sulfone and ilaprazole thiol ether has been performed using this method. 

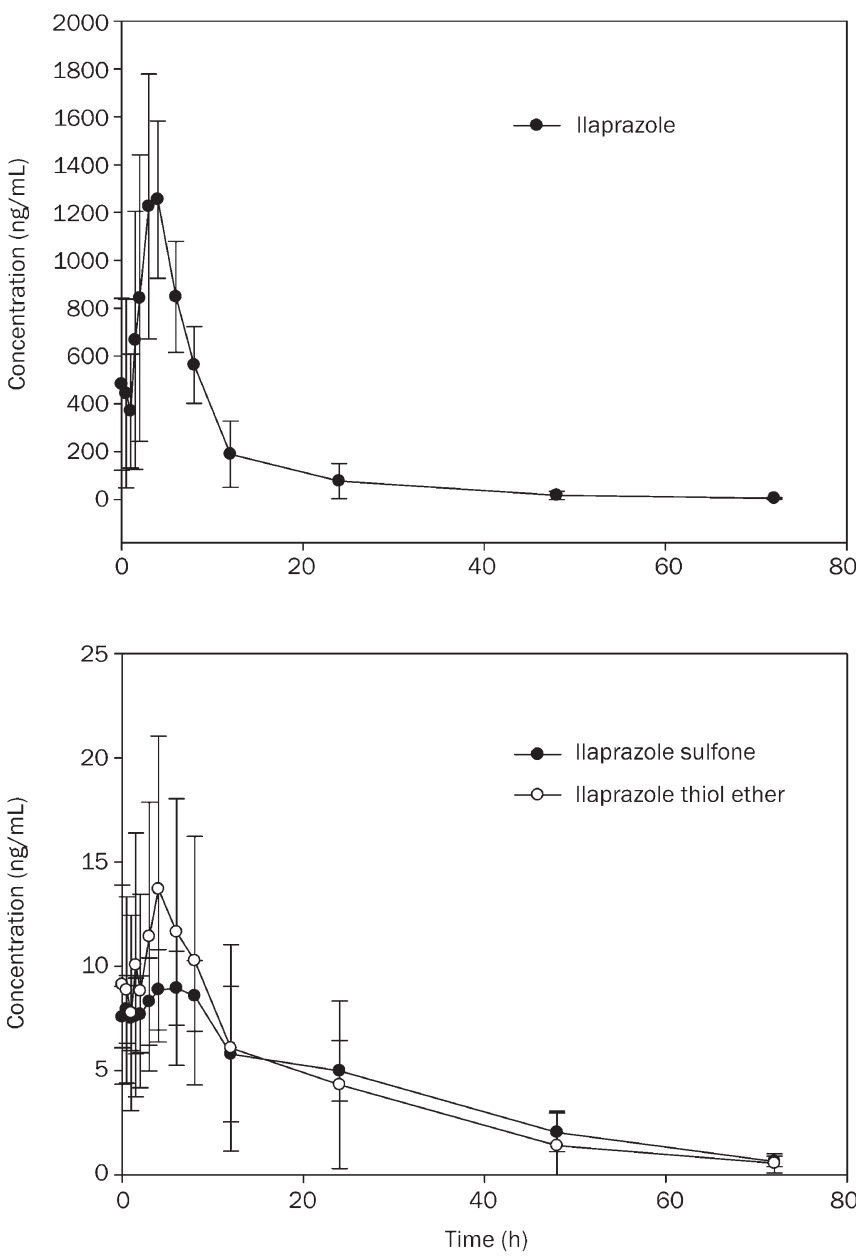

Figure 4. Mean plasma concentration vs time profile after administration of a $10 \mathrm{mg}$ oral dose of ilaprazole.

The $C_{\max }$ of ilaprazole from Figure 4 was different from the $C_{\max }$ and AUC determined in a previous study from our $\mathrm{lab}^{[4]}$. From the individual data, we found that the $C_{\max }$ values of two healthy volunteers were higher than those of the other volunteers. When we exclude the data from these two volunteers, the $C_{\max }$ and AUC values are consistent with those found in the previous study ${ }^{[6]}$. Further study of these two outlier sam- ples will be conducted by our lab.

A rapid and specific liquid chromatograph tandem mass spectrometric method has been improved and validated for the quantitative measurement of ilaprazole and its two metabolites. It has been successfully applied to characterize the pharmacokinetics of ilaprazole and its two metablites in Chinese healthy volunteers.

\section{Acknowledgements}

This work was supported by research grants from the National Natural Science Foundation of China 30528026, 30300428, 30672497, and 30500623, and by the China Medical Board of New York grants 01-755.

\section{Author contribution}

Han-wu DENG and Wei ZHANG designed the research; Zhirong TAN, Gan ZHOU, Yao CHEN, Dong GUO, and Ying-zi LIU performed the research; Dong-sheng OU-YANG analyzed data; Gan ZHOU, Zhi-rong TAN, and Lan FAN wrote the paper.

\section{References}

1 Kim EJ, Lee RK, Lee SM, Kim DY. General pharmacology of IY-81149, a new proton pump inhibitor. Arzneimittelforschung 2001; 51: 51-9.

2 Periclou AP, Goldwater R, Lee SM, Park DW, Kim DY, Cho KD, et al. A comparative pharmacodynamic study of IY-81149 versus omeprazole in patients with gastroesophageal reflux disease. Clin Pharmacol Ther 2000; 68: 304-11.

3 Myung SW, Min HK, Jin C, Kim M, Lee SM, Chung GJ, et al. Identification of IY81149 and its metabolites in the rat plasma using the on-line HPLC/ESI mass spectrometry. Arch Pharm Res 1999; 22: 189-93.

4 Li Y, Zhang W, Guo D, Zhou G, Zhou H, Xiao Z. Pharmacokinetics of the new proton pump inhibitor ilaprazole in Chinese healthy subjects in relation to CYP3A5 and CYP2C19 genotypes. Clin Chim Acta 2008; 39: 60-7.

5 Taylor PJ. Matrix effects: the Achilles heel of quantitative highperformance liquid chromatography-electrospray-tandem mass spectrometry. Clin Biochem 2005; 38: 328-34.

6 Karnes HT, March C. Precision, accuracy, and data acceptance criteria in biopharmaceutical analysis. Pharm Res 1993; 10: 1420-6.

7 Food and Drug Administration. Guidance for Industry on Bioanalytical Method Validation. Fed Regist 2001; 66: 28526-7.

8 Shah VP, Midha KK, Findlay JW, Hill HM, Hulse JD, McGilveray IJ, et al. Bioanalytical method validation - a revisit with a decade of progress. Pharm Res 2000; 17: 1551-7. 